\title{
Tubercular lesion of the foot presenting as epithelioma
}

\author{
Sarada Prasanna Sahoo ${ }^{1}$, MS, FMAS, Jagadananda Misra ${ }^{1}$, MS, B Swagat Kumar $\underline{\text { Subudhi }}{ }^{1}$, MS, \\ Abinash Kumar Panda ${ }^{1}$, Ms
}

\begin{abstract}
Tuberculosis continues to be the biggest public health problem worldwide. Tuberculosis verrucosa cutis (TVC) is a verrucose form of cutaneous tuberculosis that occurs in previously sensitised individuals due to exogenous reinfection with Mycobacterium tuberculosis or Mycobacterium bovis, and it reflects good cell-mediated immunity. An elderly man presented with multiple fungating growths, which were painless but associated with mild itching and serous discharge, on the right foot for 40 years. The lesions were free from deeper structures and no inguinal lymphadenopathy was observed. A provisional diagnosis of epithelioma was made based on the features described. However, incisional biopsy indicated that the lesions were tuberculous. As part of the anti-tubercular therapy, the lesions were excised and the wounds covered with split-thickness skin graft. The patient's recovery was uneventful, with no graft loss or infection reported. This case illustrates the importance of recognising cutaneous forms of tuberculosis, especially TVC, as an important differential diagnosis of epithelioma.
\end{abstract}

Keywords: acid-fast bacillus stains, anti-tubercular therapy, epithelioma, epitheloid granuloma, tuberculosis verrucosa cutis

\section{INTRODUCTION}

Tuberculosis remains the biggest public health problem worldwide. In the era of the human immunodeficiency virus, the impact of tuberculosis further contributes to the global burden of disease. Despite the advances made in anti-tubercular therapy and the various national programmes implemented to control the spread of the disease, tuberculosis is still a dilemma in developing countries. Cutaneous forms of tuberculosis, which include tuberculosis verrucosa cutis (TVC), scrofuloderma and lupus vulgaris, ${ }^{(1)}$ are relatively uncommon. TVC is a verrucose form of cutaneous tuberculosis that occurs in previously sensitised individuals due to exogenous reinfection with Mycobacterium tuberculosis or Mycobacterium bovis. ${ }^{(2)}$

\section{CASE REPORT}

A 62-year-old male farmer, who had been walking barefoot, was admitted to our hospital with complaints of a 40-year history of fungating growths on the dorsal aspect of his right foot. The first wart-like lesion that appeared was located on the first web space, and it had gradually progressed to involve adjacent areas (Fig. 1a). The second lesion appeared on the medial aspect of the right foot four years prior to this admission (Fig. 1b), while the third lesion appeared near the ankle one year prior to admission. Although all three lesions were painless, they were associated with mild itching and serous discharge. Systemic examination of the patient revealed no abnormalities, while local examination of the lesions showed that the lesions were firm and free from deeper structures. The lesions did not bleed on touch and no inguinal lymphadenopathy was observed. Based on clinical observations, a provisional diagnosis of epithelioma was made.

Routine investigations, including chest radiography, were performed on the patient. The results were normal, except for an
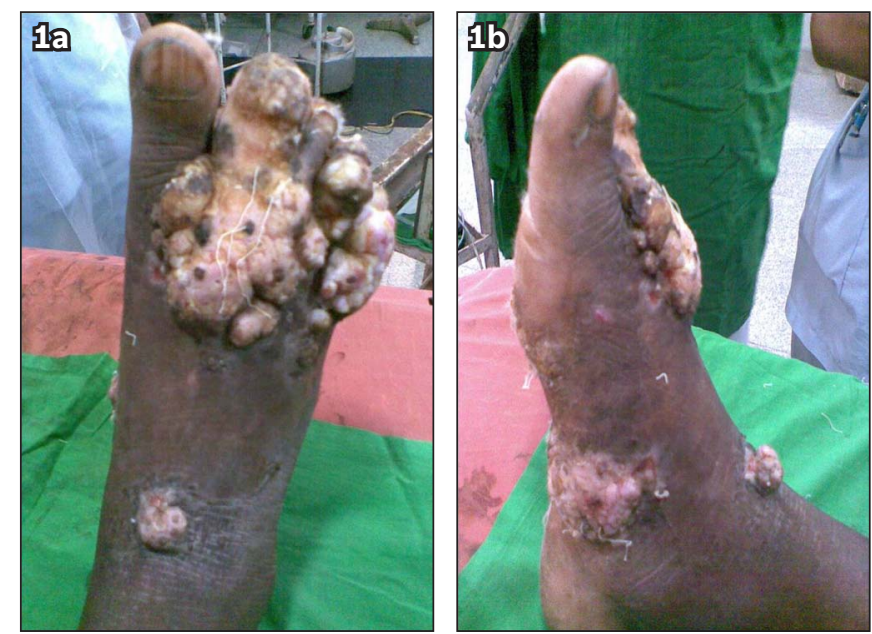

Fig. 1 Photographs show wart-like lesions in the (a) dorsal and (b) medial aspects of the patient's right foot.

elevated erythrocyte sedimentation rate (ESR) of $112 \mathrm{~mm} / 1 \mathrm{st} \mathrm{hr}$. Radiograph of the right foot did not show any bony involvement (Fig. 2). Histopathology of the incisional biopsy, however, revealed the tuberculous nature of the lesion, and the patient was investigated for signs of tuberculosis. Acid-fast bacillus (AFB) staining for sputum, Mantoux test, serum adenosine deaminase activity (ADA) and serum immunoglobulin $\mathrm{M}(\mathrm{IgM})$ for tuberculosis were all negative. Serum $\lg G$ for tuberculosis was found to be borderline. The patient was prescribed anti-tubercular therapy (ATT) category I (intensive phase: isoniazide, rifampicin, pyrazinamide and ethambutol for two months; continuation phase: isoniazide and rifampicin for four months). On completion of AAT, excision of the lesions was performed and the wounds were covered with split-thickness skin graft.

Histopathology study of the excised specimen revealed the presence of hyperplastic keratinised stratified squamous

${ }^{1}$ Department of General Surgery, Shri Ramachandra Bhanj Medical College, Cuttack, India

Correspondence: Dr Sarada Prasanna Sahoo, Senior Resident, Department of General Surgery, Shri Ramachandra Bhanj Medical College, Cuttack, Odisha 753007, India. drsarada201@gmail.com 


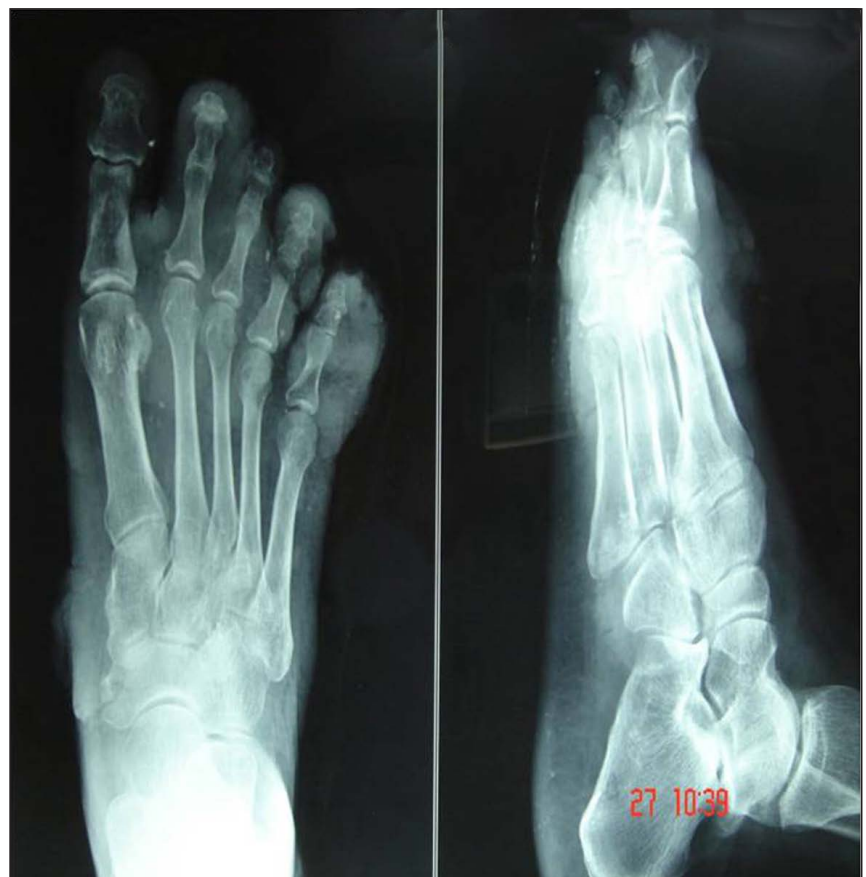

Fig. 2 Radiographs of the patient's right foot show no bony involvement.

epithelium with irregular acanthosis over fibrocollagenous stroma, as well as subepithelial tissue infiltrated with various sized granulomas containing central caseous necrosis, epitheloid cells and Langhans giant cell (Fig. 3). AFB staining of the excised specimen revealed the presence of tubercle bacilli (Fig. 4), thus confirming the diagnosis of TVC. Culture and polymerase chain reaction testing was not conducted, as the equipment for such tests was not available in our facility and the patient was unable to afford the cost of these tests. The patient recovered well with no further development of lesions at the last follow-up.

\section{DISCUSSION}

Cutaneous tuberculosis forms a small proportion of extrapulmonary tuberculosis cases. In a recently published 20-year prospective study, Masellis et al reported that $6 \%$ of all cases of cutaneous tuberculosis are TVC. ${ }^{(3)}$ Although the incidence of TVC varies according to regions of the world and from country to country, the frequency of reported cases of TVC in Asia has seen an increase in recent times. ${ }^{(4)}$ TVC lesions are most often reported in European patients' hands, while the lower limbs and buttocks are the most frequently affected sites among patients in eastern countries. ${ }^{(5)}$ In tropical climates, TVC commonly affects children who come into contact with the bacteria when sitting or walking barefooted on grounds contaminated with tuberculous sputum.

TVC may present as tumour-like lesions in the extremities. ${ }^{(5)}$ These asymptomatic lesions often occur at the sites of trauma, as seen in our patient, whose lesions were found in the foot as a result of walking barefoot. Most cases of TVC are due to exogenous reinfection in individuals with marked cutaneous hypersensitivity and good cell-mediated immunity; autoinoculation from sputum is an unlikely cause. ${ }^{(6)}$ Although

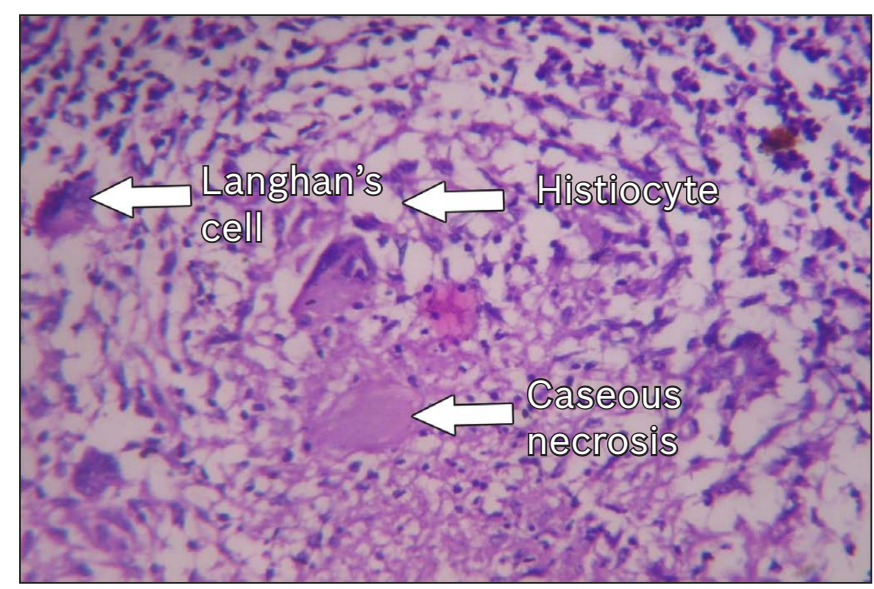

Fig. 3 Photomicrograph of the excised lesion shows Langhan's cells, histocytes and caseous necrosis, thus confirming tuberculoma. (Haematoxylin \& eosin, $\times 40$ ).

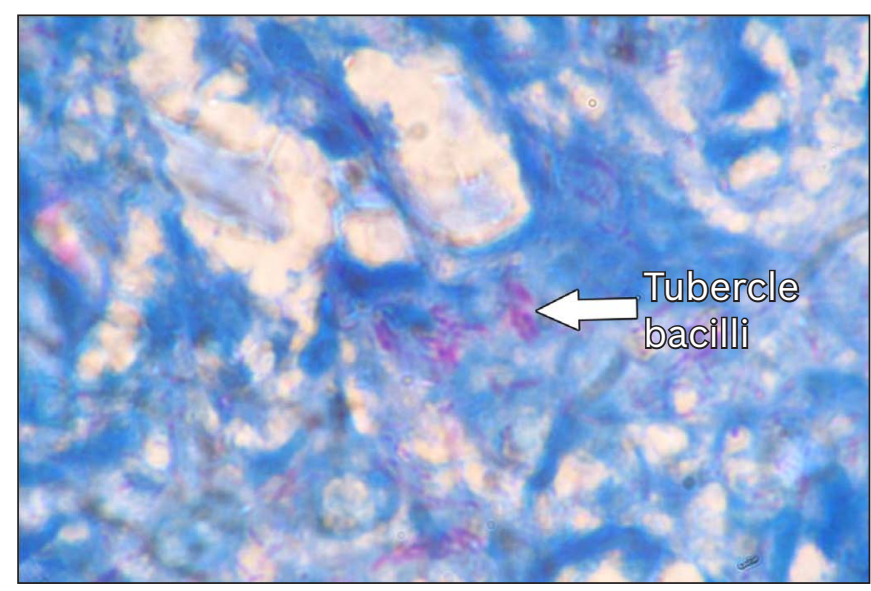

Fig. 4 Photomicrograph of the excised specimen shows the presence of tubercle bacilli (AFB staining, $\times 1,000$ ).

some incidents of TVC involving lymphadenopathy (with inguinal lymph nodes most commonly involved) have been reported,(7) lymphadenopathy is generally not associated with TVC. No correlation has been found between Mantoux positivity or previous Bacillus Calmette-Guerin vaccination and the extent of disease. ${ }^{(8)}$ In tubercular skin disease with a high level of skin sensitivity, the number of bacteria within the lesion is reportedly few. ${ }^{(9)}$ On histopathological study, the presence of granuloma is a cardinal sign of TVC, whereas a typical tuberculous focus with caseating necrosis is uncommon. The diagnosis and management of TVC are based mainly on the clinical appearance and presence of positive microscopic features in the histopathological study of the lesion.

In this report, we have shown that TVC may be erroneously diagnosed as epithelioma of the skin, as the two conditions share similar features. Histopathological examination is thus mandatory and essential for accurate diagnosis, so that an effective treatment protocol for TVC may be implemented.

\section{ACKNOWLEDGEMENT}

We are thankful to the staff of Department of Pathology, Shri Ramachandra Bhanj Medical College, Cuttack, India, for the help rendered in the writing of this case report. 


\section{REFERENCES}

1. Puri N. A clinical and histopathological profile of patients with cutaneous tuberculosis. Indian J Dermatol 2011; 56:550-2.

2. Wolff K, Tappeiner G. Mycobacterial diseases: tuberculosis and atypical mycobacterial infections. Vol 2. In: Freedberg IM, Eisen AZ, Katz SI, et al, eds. Fitzpatrick's Dermatology in General Medicine. 5th ed. New York: McGraw-Hill, 1999: 2152-80.

3. Masellis P, Gasparini G, Caputo R, Alessi E. Tuberculosis verrucosa cutis which remained undiagnosed for forty-three years. Dermatology 1995; 191:145-8.

4. Pereira MB, Gomes MK, Pereira F. Tuberculosis verrucosa cutis associated with tuberculous lymphadenitis. Int J Dermatol 2000; 39:856-8

5. lizawa O, Aiba S, Tagami H. Tuberculosis verrucosa cutis in a tumour-like form. Br J Dermatol 1991; 125:79-80

6. Aliağaoğlu C, Atasoy M, Güleç Ai, et al. Tuberculosis verrucosa cutis. Eur J Gen Med 2009; 6:268-73.

7. Kakakhel KU, Fritsch P. Cutaneous tuberculosis. Int J Dermatol 1989; 28:355-62.

8. Kumar B, Kaur S. Pattern of cutaneous tuberculosis in North India. Indian J Dermatol Venereol Leprol 1986; 52:203-7.

9. Lantos G, Fisher BK, Contreras M. Tuberculous ulcer of the skin. J Am Acad Dermatol 1988; 19:1067-72. 